Research Paper

\title{
Short Survival Time after Palliative whole Brain Radiotherapy: Can We Predict Potential Overtreatment by Use of a Nomogram?
}

\author{
Carsten Nieder ${ }^{1,2}$, Jan Norum², 3, Mandy Hintz ${ }^{4}$, Anca L. Grosu ${ }^{4,5 凶}$ \\ 1. Department of Oncology and Palliative Medicine, Nordland Hospital, 8092 Bodø, Norway; \\ 2. Department of Clinical Medicine, Faculty of Health Sciences, University of Troms $ø, 9037$ Troms $\varnothing$, Norway; \\ 3. Northern Norway Regional Health Authority trust, 8038 Bodø, Norway; \\ 4. Department of Radiation Oncology, University Hospital Freiburg, 79106 Freiburg, Germany; \\ 5. German Cancer Consortium (DKTK), Freiburg, and German Cancer Research Centre (DKFZ), D-69121 Heidelberg, Germany.
}

$\triangle$ Corresponding author: Carsten Nieder, MD, Department of Oncology and Palliative Medicine, Nordland Hospital, 8092 Bodø, Norway, Tel: +47 75578449 , FAX: +47 7553 4975, e-mail: carsten.nieder@nlsh.no

(C) Ivyspring International Publisher. This is an open access article distributed under the terms of the Creative Commons Attribution (CC BY-NC) license (https://creativecommons.org/licenses/by-nc/4.0/). See http://ivyspring.com/terms for full terms and conditions.

Received: 2016.12.02; Accepted: 2017.02.04; Published: 2017.06.01

\begin{abstract}
Background: Many patients with brain metastases undergoing whole brain radiotherapy (WBRT) have very limited survival. The purpose of this study was to validate a nomogram derived from a large American database and to examine its ability to better predict short survival (cut-off 2 months) than previous models.

Material and Methods: This retrospective study included 254 European patients treated with primary WBRT. In addition, an exploratory analysis of patients managed with best supportive care (BSC) was performed too.

Results: Median survival after WBRT was 3.0 months. The median nomogram point sum was 122 (range $31-212$ ). The nomogram-predicted median survival for a patient with 122 points is 3.3 months. Despite the nomogram's ability to stratify the patients into different prognostic groups, the survival curves of patients with intermediate point sum in the range of 90-139 points were largely superimposable. The poorest prognostic group with $\geq 180$ points had a median and maximum survival of 1.8 and 4.6 months, respectively. Among these 18 patients $(7 \%) 9$ survived for less than and 9 for more than 2 months. Comparable survival outcomes were observed after BSC in a smaller group of 8 patients with $\geq 180$ points.

Conclusions: Because of several differences between the original and validation findings, the nomogram should be examined in additional large databases. Its ability to predict poor survival is promising and possibly comparable to our previously published models. The final goal of developing a validated model that allows poor prognosis patients to safely forego WBRT without compromising survival or quality of life requires further research efforts.
\end{abstract}

Key words: brain metastases, prognostic factors, nomogram, radiotherapy.

\section{Introduction}

Development of better local and systemic treatment approaches has resulted in individually tailored management of patients with parenchymal brain metastases from solid extracranial primary tumors [1]. High local control rates and preservation of neurocognitive function can be achieved with stereotactic radiotherapy and surgical resection [2]. These recent developments have led to a decrease in the prescription of primary whole brain radiotherapy (WBRT). Fewer and fewer patients with favorable prognostic features are now being treated with upfront WBRT as the sole local management 
approach [3]. However, it is a matter of debate whether or not WBRT should be offered to patients with adverse prognostic factors [4]. Their survival is often limited to few weeks, and therefore best supportive care (BSC) might also be an option. The challenge is, however, to predict who is in this subgroup. Previous attempts to use prognostic scores, such as the recursive partitioning analysis (RPA) [5] or graded prognostic assessment (GPA) [6], to select patients appropriate for BSC were not successful [7, 8]. Even within the poor prognosis group of the RPA or diagnosis-specific (DS) GPA survival was heterogeneous. Thus, clinicians are afraid to withhold local therapy to patients who may experience prolonged survival when local measures lead to, at least temporary, control of the brain metastases. A disadvantage of this practice is overtreatment of patients with short survival, resulting in the fact that they often spend a large part of the remaining life span on active treatment [9] instead of spending time with their beloved ones.

Barnholtz-Sloan et al. [10] developed a nomogram for individualized estimation of overall survival from the Radiation Therapy Oncology Group's (RTOG) randomized trials [10]. The overall median survival in this large study with more than 2000 patients was 136 days (4.5 months). Table 1 shows the baseline characteristics that contributed to the nomogram. They were a mixture of the prognostic factors that also were part of the RPA, GPA and DS-GPA. In this model, the use of the nomogram results in a total point sum that corresponds to a certain predicted median, 6-month and 12-month survival probability. The purpose of the present study was to validate the nomogram in a European patient population and to examine its ability to predict short time survival better than the models previously tested by our group. We used identical methods and definitions as in our previous studies, e.g. for short survival (max. 2 months) [7].

Table 1. Baseline characteristics included in the nomogram (Barnholtz-Sloan et al. 2012 [8])

\begin{tabular}{llc}
\hline Parameter & \multicolumn{2}{c}{ Also included in the RPA and } \\
& DS-GPA & \\
\hline $\begin{array}{l}\text { Primary tumor site and histology } \\
\text { Status of primary disease }\end{array}$ & no & yes \\
(controlled/uncontrolled) & & no \\
Metastatic spread (brain alone/other sides) & yes & yes \\
Age & yes & yes \\
Karnofsky performance status & yes & yes \\
Number of brain lesions (single/multiple) & no & yes \\
\hline RPA: recursive partitioning analysis & & \\
DS-GPA: diagnosis-specific graded prognostic assessment &
\end{tabular}

DS-GPA: diagnosis-specific graded prognostic assessment

\section{Material and Methods}

\section{Patients and treatment}

This retrospective intention-to-treat study included 254 consecutive patients with brain metastases from common primary tumors included in the DS-GPA model (breast, lung, kidney, gastrointestinal cancer and malignant melanoma) who received palliative WBRT as primary treatment. Total doses in the range of 20-40 Gy were prescribed. The number of fractions ranged from 5-20. Patients who failed to complete all fractions of WBRT were also included. Since the main endpoint was short time survival, patients treated with upfront resection or stereotactic radiotherapy were not included, as those were supposed to have a superior prognosis. Most institutions reserve aggressive local management for patients considered to have better prognosis. The patients were treated between 2006 and 2015 and identified from a previously described prospectively maintained database [11], which included data from the radiotherapy centers in Bodø and Freiburg. Second line surgery or stereotactic radiotherapy for failure after WBRT was not an exclusion criterion. Systemic treatment was given according to local standards at the discretion of the medical oncologists. Prognosis was calculated according to the RPA, DS-GPA and nomogram as described in the original publications $[5,6,10]$.

\section{Statistical methods}

Actuarial survival from the first day of radiotherapy was calculated employing the Kaplan-Meier method, and different groups were compared using the log-rank test (SPSS 22, IBM Corp., Armonk, NY, USA). Nine patients were alive at last follow-up (May 15, 2016). The median follow-up was 22 months (range 4-84). Date of death was known in all other patients.

\section{Results}

\section{Patient characteristics}

The median age was 64 years (range 24-93). The median Karnofsky performance status (KPS) was 70 (range 30-100). Ninety-nine patients (39\%) had less than four brain metastases. A controlled primary tumor was present in 164 patients (65\%). However 220 had extracranial metastases (87\%). Eighty-eight patients (35\%) had non-small cell lung and 61 (24\%) breast cancer. Further patient characteristics are shown in Table 2. According to the RPA score, 10 patients (4\%) had a favorable, 150 (59\%) an intermediate, and 94 (37\%) an unfavorable prognosis. According to the 4-tiered DS-GPA model, 9 patients 
(3\%) were in the best, 34 (13\%) in the second best, 64 $(25 \%)$ in the second worst, and $146(57 \%)$ in the worst prognostic group. The median DS-GPA point sum was 1.0 .

Table 2. Primary tumor type and nomogram point sum

\begin{tabular}{llll}
\hline Primary & $\mathrm{n}$ & $\begin{array}{l}\text { Median sum } \\
\text { (range) }\end{array}$ & $\begin{array}{l}\text { Median survival in } \\
\text { months }\end{array}$ \\
\hline $\begin{array}{l}\text { Breast cancer } \\
\text { NSCLC, }\end{array}$ & 61 & $83(31-131)$ & 4.0 \\
$\begin{array}{l}\text { adenocarcinoma } \\
\text { NSCLC, squamous cell }\end{array}$ & 14 & $119(47-192)$ & 3.5 \\
$\begin{array}{l}\text { carcinoma } \\
\text { NSCLC, large cell }\end{array}$ & 12 & $135.5(135-201)$ & 2.4 \\
$\begin{array}{l}\text { carcinoma } \\
\text { SCLC }\end{array}$ & 27 & $147.5(54-200)$ & 3.2 \\
$\begin{array}{l}\text { Malignant melanoma } \\
\text { Gastrointestinal tumor* }\end{array}$ & 35 & $140(74-202)$ & 3.0 \\
Kidney cancer & 17 & $146.5(79-212)$ & 2.0 \\
\hline
\end{tabular}

NSCLC: non-small cell lung cancer, SCLC: small cell lung cancer

*mainly colorectal and esophageal cancer

\section{RPA and DS-GPA}

Both scores predicted survival. According to the three-tiered RPA, median survival was 5.6, 3.8 and 1.9 months $(p<0.0001$, log-rank test pooled over all strata). In class III 6-month and 12-month survival rates were 12 and $4 \%$, respectively. According to the four-tiered DS-GPA, median survival was 16.4, 4.5, 3.5 and 2.5 months $(p<0.0001$, log-rank test pooled over all strata). In class IV 6-month and 12-month survival rates were 17 and $8 \%$, respectively.

\section{Nomogram and survival}

The median nomogram point sum was 122 (range $31-212,25^{\text {th }}$ percentile $94,75^{\text {th }}$ percentile 151 ). The median survival was 3.0 months $(95 \%$ confidence interval 2.6-3.4 months). The nomogram-predicted median survival for a patient with 122 points is 100 days (3.3 months). Table 3 shows survival outcomes for different prognostic groups with median survival ranging from 1.8 to 14.8 months for the extreme strata, defined as a point sum of $<70$ points and $\geq 180$ points, respectively $(p<0.0001$, log-rank test pooled over all strata). Despite this ability to stratify the patients into different prognostic groups, the survival curves of patients with intermediate point sum, especially in the range of 90-139 points, were superimposable, as already suggested by the data shown in Table 3 . Figure 1 shows the comparison of our own and RTOG database-derived survival outcomes. For many of the strata, minimal differences were observed. The poorest prognostic group with $\geq 180$ points had a median and maximum survival of 1.8 and 4.6 months, respectively. Among these 18 patients (7\%), 9 survived for less than and 9 for more than 2 months.
We observed survival of less than 2 months in one patient with point sum $<70(1 / 21,5 \%)$, three patients with point sum $70-79(3 / 16,19 \%)$, and four patients with point sum $80-89(4 / 17,24 \%)$.

Table 3. Survival outcomes stratified by nomogram point sum $(p<0.0001$, log-rank test pooled over all strata)

\begin{tabular}{lllll}
\hline $\begin{array}{l}\text { Point } \\
\text { sum }\end{array}$ & $\mathrm{n}$ & $\begin{array}{l}\text { Median survival } \\
\text { in months }\end{array}$ & $\begin{array}{l}\text { 6-month survival } \\
\text { probability }\end{array}$ & $\begin{array}{l}\text { 12-month survival } \\
\text { probability }\end{array}$ \\
\hline$<70$ & 21 & 14.8 & $71 \%$ & $52 \%$ \\
$70-79$ & 16 & 3.1 & $38 \%$ & $19 \%$ \\
$80-89$ & 17 & 3.5 & $41 \%$ & $12 \%$ \\
$90-99$ & 23 & 4.0 & $24 \%$ & $9 \%$ \\
$100-109$ & 24 & 3.1 & $25 \%$ & $8 \%$ \\
$110-119$ & 21 & 3.6 & $24 \%$ & $13 \%$ \\
$120-129$ & 23 & 2.6 & $22 \%$ & $17 \%$ \\
$130-139$ & 20 & 3.0 & $30 \%$ & $25 \%$ \\
$140-149$ & 23 & 2.4 & $22 \%$ & $13 \%$ \\
$150-159$ & 25 & 2.0 & $24 \%$ & $4 \%$ \\
$160-169$ & 13 & 2.5 & $15 \%$ & $0 \%$ \\
$170-179$ & 10 & 2.8 & $0 \%$ & $0 \%$ \\
$\geq 180$ & 18 & 1.8 & $0 \%$ & $0 \%$ \\
\hline
\end{tabular}

\section{Best supportive care (BSC)}

Our brain metastasis database contained 51 patients managed with BSC who had complete information to calculate the nomogram point sum. Eight of these $(16 \%)$ had a point sum $\geq 180$. Median and maximum survival were 1.6 and 4.2 months, respectively. Four of them survived for more than 2 months.

\section{Discussion}

We performed a retrospective validation study of the nomogram developed by Barnholtz-Sloan et al. [10] in a European patient population and examined the nomogram's ability to predict short time survival better than previous models tested by our group $[7,8]$. The study population consisted mainly of patients with intermediate or poor prognosis who were judged not to be appropriate candidates for aggressive local therapies, such as surgery or stereotactic radiotherapy. In the RTOG database, a small minority of resected or radiosurgically treated patients were included, e.g., participants in RTOG trial 95-08, while the vast majority was treated with primary palliative WBRT [10]. In addition, $57 \%$ of our patients belonged to the worst prognostic DS-GPA group, compared to $11 \%$ in the original study. This discrepancy likely explains why the median survival in our study was only 3.0 months, while the RTOG patients survived for a median of 4.5 months. The prognostic impact of both RPA and DS-GPA could be confirmed in our study. 


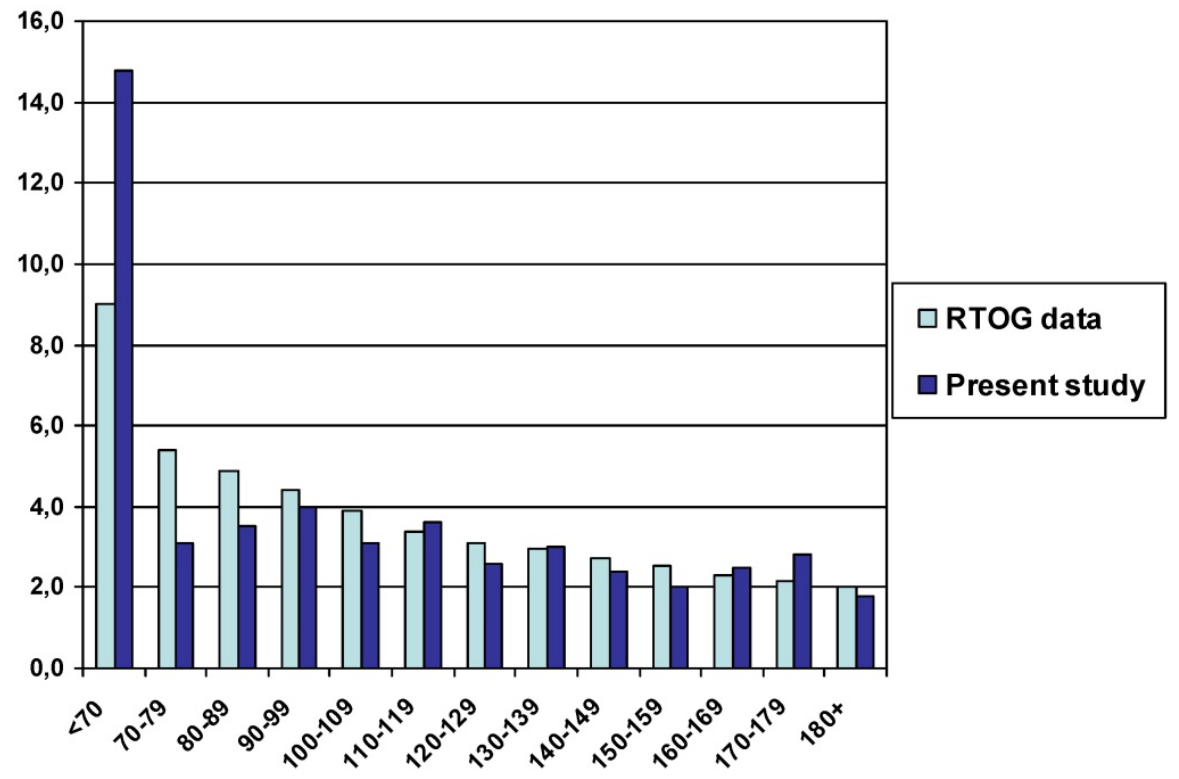

Nomogram point sum

Figure 1. Median survival of patients with different point sums in the RTOG and own database

Limitations of this study include the number of patients, statistical power of subgroup analyses, and retrospective design. Whereas the number of patients was limited in our study database, they represent the total cohort of the two radiotherapy departments and consequently express the daily practice at these units. It is possible that the nomogram results would have matched the RTOG database findings even closer in a larger cohort of patients. However, we could confirm that the nomogram predicts especially favorable and unfavorable survival with high accuracy. A certain proportion of false positive and false negative findings is a known drawback of essentially all predictive tools available in the field of oncology [12-15]. For patients with a point sum in the order of 90-139 points, no clear survival differences emerged. It is therefore necessary to examine the nomogram in additional databases.

A secondary endpoint of our study was prediction of very short survival after WBRT. In the terminal phase of cancer progression, many patients might not survive long enough to experience the beneficial effects of radiotherapy [16]. Disadvantages to patients and family members, such as acute side effects, travel distance and out-of-pocket costs, must also be considered. Similarly, unnecessary use of specialized health care resources (e.g., radiotherapy) should be kept in mind as such resources may be allocated to other patients and waiting lists may consequently be further minimized. It would therefore be desirable to avoid fruitless WBRT in patients who may experience the same survival and quality of life with optimal BSC. We have previously reported that patients with a DS-GPA score of 0-1.5 points and age $\geq 75$ years or KPS $\leq 50$ or uncontrolled primary tumor with extracranial metastases to at least two organs might be appropriate candidates for BSC [7]. A follow-up study suggested that patients with a combination of three new adverse features (elevated serum lactate dehydrogenase plus low albumin plus extracranial metastases to at least two organs) might also be considered for BSC [17]. We hoped that the nomogram could be an even better tool, as it is easy to handle and accurate enough to predict short survival of 2 months or less. However, even among those $7 \%$ of patients with the highest point sums $(\geq 180), 50 \%$ survived for 2-4.6 months. Our BSC database was too small to create a sufficiently large group of patients with $\geq 180$ points managed without WBRT. However, it was interesting to note, from this exploratory analysis, that the small group of 8 patients had survival outcomes that resembled those of their WBRT counterparts very closely. Despite the promising potential of the nomogram, our previous recommendations for patient selection will remain unaltered until larger studies have confirmed that WBRT and BSC result in undistinguishable outcomes.

As already realized in the RPA studies [5], patients with poor KPS (RPA class 3) are a heterogeneous group. Despite short median survival, post-treatment functional improvement is possible and might even be associated with survival beyond 12 months. Lee et al. have also reported that RPA class 3 patients who presented with hemiparesis, cognitive deficit or apraxia could respond to palliative radiotherapy with the potential to benefit from 
progression-free survival of 12 months or more [18], a finding in line with the authors' personal experience. In a prospective randomized phase II study WBRT was associated with improvements in cognitive and emotional function but also with worsened nausea [19]. Complete and partial imaging response rate was $55 \%$. In a different study, a total of 14 symptom scores and overall quality of life were collected prospectively in 217 patients for up to 3 months [20]. Appetite loss, weakness, and nausea significantly increased from baseline, while balance, headache, and anxiety significantly decreased from baseline. Clearly, quality of life is a complex endpoint, which is influenced by extracranial disease extent and also side effects of systemic therapy. This endpoint has not been evaluated in our study. In general, important goals for the treatment of brain metastases include alleviating neurological symptoms, preventing their progression and neurological deterioration, and thus enhancing quality of life. Treatment decisions should not be based on life expectancy alone.

\section{Conclusions}

Because of several differences between RTOG database and present results, the nomogram should be examined in additional large studies. Despite its general ability to predict long and short time survival, our previous models for poor prognosis patients appear preferable because they were derived from larger subgroups. The final goal of developing a validated model that allows patients with adverse prognostic features to safely forego WBRT without compromising survival, symptom control and quality of life requires further research efforts.

\section{Competing Interests}

The authors have declared that no competing interest exists.

\section{References}

1. Bertolini F, Spallanzani A, Fontana A, et al. Brain metastases: an overview. CNS Oncol. 2015; 4: 37-46.

2. Leth T, von Oettingen G, Lassen-Ramshad YA, et al. Survival and prognostic factors in patients treated with stereotactic radiotherapy for brain metastases. Acta Oncol. 2015; 54: 107-14.

3. Hendriks LE, Troost EG, Steward A, et al. Patient selection for whole brain radiotherapy (WBRT) in a large lung cancer cohort: Impact of a new Dutch guideline on brain metastases. Acta Oncol. 2014; 53: 945-51.

4. Nieder C, Norum J, Dalhaug A, et al. Radiotherapy versus best supportive care in patients with brain metastases and adverse prognostic factors. Clin Exp Metastasis. 2013; 30: 723-9.

5. Gaspar LE, Scott C, Murray K, Curran W. Validation of the RTOG recursive partitioning analysis (RPA) classification for brain metastases. Int J Radiat Oncol Biol Phys. 2000; 47: 1001-6.

6. Sperduto PW, Chao ST, Sneed PK, et al. Diagnosis-specific prognostic factors, indexes, and treatment outcomes for patients with newly diagnosed brain metastases: a multi-institutional analysis of 4,259 patients. Int J Radiat Oncol Biol Phys. 2010; 77: 655-61.

7. Nieder C, Pawinski A, Molls M. Prediction of short survival in patients with brain metastases based on three different scores: a role for 'triple-negative' status? Clin Oncol (R Coll Radiol). 2010; 22: 65-9.
8. Nieder C, Norum J, Dalhaug A, et al. Best supportive care in patients with brain metastases and adverse prognostic factors: development of improved decision aids. Support Care Cancer. 2013; 21: 2671-8.

9. Gripp S, Mjartan S, Boelke E, Willers R. Palliative radiotherapy tailored to life expectancy in end-stage cancer patients: reality or myth? Cancer. 2010; 116: 3251-6.

10. Barnholtz-Sloan JS, Yu C, Sloan AE, et al. A nomogram for individualized estimation of survival among patients with brain metastasis. Neuro Oncol. 2012; 14: 910-8.

11. Nieder C, Hintz M, Grosu AL. Predicted survival in patients with brain metastases from colorectal cancer: Is a current nomogram helpful? Clin Neurol Neurosurg. 2016; 143: 107-10.

12. Lambin P, van Stiphout RG, Starmans $M H$, et al. Predicting outcomes in radiation oncology--multifactorial decision support systems. Nat Rev Clin Oncol. 2013; 10: 27-40.

13. Gray EP, Teare MD, Stevens J, Archer R. Risk prediction models for lung cancer: A systematic review. Clin Lung Cancer. 2016; 17: 95-106.

14. Oh YJ, Yi SY, Kim KH, et al. Prognostic model to predict survival outcome for curatively resected liposarcoma: A multi-institutional experience. J Cancer. 2016; 7: 1174-80.

15. Kluth LA, Black PC, Bochner $\mathrm{BH}$, et al. Prognostic and prediction tools in bladder cancer: A comprehensive review of the literature. Eur Urol. 2015; 68: 238-53.

16. Lutz ST, Jones J, Chow E. Role of radiation therapy in palliative care of the patient with cancer. J Clin Oncol. 2014; 32: 2913-9.

17. Nieder C, Marienhagen K, Dalhaug A, et al. Prognostic models predicting survival of patients with brain metastases: integration of lactate dehydrogenase, albumin and extracranial organ involvement. Clin Oncol ( $R$ Coll Radiol). 2014; 26: 447-52.

18. Lee SH, Lee KC, Choi J, et al. Clinical application of RapidArc volumetric modulated arc therapy as a component in whole brain radiation therapy for poor prognostic, four or more multiple brain metastases. Radiat Oncol J. 2012; 30: 53-61.

19. Rojas-Puentes LL, Gonzalez-Pinedo M, Crismatt A, et al. Phase II randomized, double-blind, placebo-controlled study of whole-brain irradiation with concomitant chloroquine for brain metastases. Radiat Oncol. 2013; 8: 209.

20. Wong E, Zhang L, Rowbottom L, et al. Symptoms and quality of life in patients with brain metastases receiving whole-brain radiation therapy. Support Care Cancer. 2016; 24: 4747-59. 